\title{
Fatores de risco cardiovascular do Escore de Framingham entre hipertensos assistidos por equipes de Saúde da Família
}

Cardiovascular risk factors on the Framingham Risk Score among hypertensive patients attended by family health teams

Antônio Prates Caldeira ${ }^{1}$

${ }^{1}$ Departamento de Saúde da Mulher e da Criança, Universidade Estadual de Montes Claros. Campus Universitário Professor Darcy Ribeiro. 39.401-089 Montes Claros MG Brasil. antonio.caldeira@unimontes.br
Abstract The Framingham Risk Score (FRS) is used to stratify cardiovascular risk. This study sought to identify the prevalence of risk factors used in the FRS and the use of drugs that reduce cardiovascular risk among hypertensive patients attended by the Family Health Strategy (FHS). It is cross-sectional study, which evaluated a random sample of hypertensive patients in the FHS in northern Minas Gerais. Data were collected through interviews and from medical records. 505 hypertensive patients were evaluated in 9 municipalities of the region, with a predominance of women; 325 (64.4\%), mean age of 66.4 years. In 90\% of hypertensive patients there was at least one associated risk factor. The grouping of three or more factors occurred in 79 (15.7\%) patients. Dyslipidemia was found in 188 (37.2\%), diabetes in 101 (20\%) and smoking in 36 (7.1\%). The use of anti-hypertensive drugs was observed in 481 (95.2\%) patients. The use of other cardioprotective drugs was higher among patients with 3 or more risk factors: $45.6 \%$ used antiplatelet and $27.8 \%$ used lipid-lowering drugs. The prevalence of risk factors from the FRS in the population studied was quite high and the use of cardioprotective drugs was seen to be restricted. Strategies to increase the use of FRS may improve the management of hypertension in the FHS.

Key words Hypertension, Risk factors, Primary health care
Resumo O Escore de Framingham (EF) é utilizado para estratificar o risco cardiovascular. $O$ presente estudo objetivou identificar, entre os hipertensos assistidos pela Estratégia Saúde da Família (ESF), a prevalência dos fatores de risco utilizados no EF e do uso de drogas que reduzem o risco cardiovascular. Trata-se de estudo transversal, que avaliou amostra aleatória de hipertensos da ESF no norte de Minas Gerais. Os dados foram coletados em entrevistas e consulta aos prontuários. Foram avaliados 505 hipertensos em $9 \mathrm{mu}$ nicípios da região, com predomínio de mulheres, 325 (64,4\%) e média de idade de 66,4 anos. Em 90\% dos hipertensos houve pelo menos 1 fator de risco associado. O agrupamento de 3 ou mais fatores ocorreu em 79 (15,7\%) pacientes. Dislipidemia foi encontrada em 188 (37,2\%), diabetes em 101 (20\%) e tabagismo em 36 (7,1\%). O uso de anti-hipetensivo foi observado em 481 (95,2\%) hipertensos. $O$ uso de outras drogas cardioprotetoras foi maior entre pacientes 3 ou mais fatores de risco: 45,6\% usavam antiagregantes plaquetários e 27,8\% usavam hipolipemiantes. A prevalência de fatores de risco do EF na população estudada mostrou-se bastante elevada e o uso de drogas cardioprotetoras mostrou-se restrito. Estratégias para ampliar o uso do EF podem melhorar o manejo da hipertensão arterial na ESF.

Palavras-chave Hipertensão, Fatores de risco, Atenção Primária à Saúde 


\section{Introdução}

A hipertensão arterial sistêmica (HAS) é o principal fator de risco para as doenças cardiovasculares (DCV), sendo uma condição clínica associada à elevada mortalidade ${ }^{1}$. Salienta-se ainda que cerca de $80 \%$ dos hipertensos possuem comorbidades como diabetes, dislipidemia, tabagismo ou história familiar de ateromatose ${ }^{2}$. A simultaneidade de fatores de risco cardiovascular mostrouse comum em estudo realizado no sudeste brasileiro ${ }^{3}$. Um aspecto relevante desse fato é que a combinação destes fatores de risco para o desenvolvimento de DCV é maior do que a soma dos mesmos tomados isoladamente ${ }^{4}$. Assim, a abordagem do hipertenso deve levar em conta características de cada indivíduo, como coexistência de outros fatores de risco e lesões de órgãos-alvo ${ }^{5}$.

Para essa abordagem individualizada é necessária a estratificação do hipertenso baseando-se em níveis pressóricos e fatores de risco associados. Essa estratificação é uma maneira de calcular o risco cardiovascular global e projetar o risco de um indivíduo ao longo do tempo, com vistas à redução da mortalidade por DCV, mais do que simplesmente adequação dos níveis pressóricos. É também uma forma compreensível de explicar ao paciente como a adesão ao tratamento pode reduzir o risco cardiovascular ${ }^{2}$.

O Ministério da Saúde propõe a utilização da estratificação para definir o prognóstico e a conduta na abordagem do hipertenso na atenção primária $^{6}$, recomendando a adoção do Escore de Framingham $(\mathrm{EF})^{7}$, que tem sido testado com sucesso em várias populações ${ }^{8,9}$. Trata-se de uma escala que busca estimar o risco de um evento cardiovascular para o paciente, a partir de algumas variáveis. Considerando que os fatores de risco utilizados nessa estratificação são frequentes no Brasil ${ }^{3,10,11}$, sua utilização tem potencial para melhorar o controle do portador de HAS no país e reduzir a ocorrência de desfechos graves para os mesmos.

Em princípio, a grande maioria dos pacientes hipertensos deve ser assistida ao nível da atenção primária por meio de equipes de Saúde da Família. Infelizmente, alguns estudos têm revelado grande fragilidade de tais equipes no manejo do paciente hipertenso $\mathrm{o}^{12,13}$. Nesse sentido a utilização do EF representa uma abordagem inovadora com potencial de ampliar a adesão ao tratamento e nortear resultados mais efetivos.

No presente estudo, objetivou-se identificar, entre os hipertensos assistidos por equipes de Saúde da Família, a prevalência dos fatores de risco utilizados no escore de Framingham e do uso de drogas que reduzem o risco cardiovascular.

\section{Métodos}

Trata-se de um estudo transversal, realizado em pacientes selecionados aleatoriamente a partir da ficha de cadastros de hipertensos (ficha "B") do Sistema de Informações da Atenção Básica (SIAB) das equipes de saúde de família (ESF) da área urbana em municípios do norte de Minas Gerais.

O tamanho da amostra foi calculado considerando-se a população estimada de 1.500 .000 habitantes para a região (dados do $\mathrm{IBGE}^{14}$ ) e uma prevalência estimada de HAS de 25\%. Adotou-se um nível de confiança de $95 \%$ e erro estimado de $5 \%$, obtendo-se o valor de 288 pacientes para uma amostragem aleatória simples. Considerando-se a alocação das ESF como amostragem por conglomerados, multiplicou-se o número encontrado por 1,5 (fator de correção) e acrescentouse $15 \%$ a este valor prevendo-se perdas. Desta maneira a amostra final mínima calculada foi de 497 indivíduos.

A seleção dos pacientes foi definida em dois estágios. No primeiro, selecionou-se por técnica aleatória simples, os municípios e as equipes de Saúde da Família. Neste estágio, os municípios com maior número de equipes da ESF tiveram maiores chances de serem sorteados. No segundo estágio, a partir do cadastro dos hipertensos nestas equipes, foram selecionados por amostragem aleatória simples os indivíduos para o estudo. Foram excluídos os pacientes que informaram ou que tinham registro no prontuário de evento cardiovascular prévio (infarto agudo do miocárdico, angina pectoris, acidente vascular encefálico, doença arterial periférica ou insuficiência cardíaca) visto que o EF é preconizado para ser uma ferramenta para prevenção primária. Foram alocados apenas hipertensos acima de 40 anos de idade, pois é a partir desta faixa etária que a estratificação de risco tem uma maior da aplicabilidade ${ }^{15}$. No processo de seleção em cada equipe de saúde, quando o indivíduo sorteado não tinha idade superior a 40 anos era substituído por outro. Por questões logísticas foram excluídos os pacientes residentes em zonas rurais.

Os dados foram coletados por meio de entrevistas aos pacientes e consulta aos prontuários, como estratégia para confirmar informações coletadas. Quando havia discordância entre as informações do paciente e do prontuário, estas últimas eram tomadas como verdadeiras. Utili- 
zou-se um formulário padronizado, com questões fechadas, que visavam obter informações demográficas e identificação de fatores de risco utilizados no EF. Os fatores identificados foram: idade, sexo, presença de dislipidemia, diabetes e tabagismo atual. Também foram obtidas informações sobre fatores de risco concomitantes, como sedentarismo (considerando-se não sedentário o indivíduo que praticava atividades físicas regularmente, pelo menos 30 minutos por dia na maioria dos dias da semana ${ }^{16}$ ) e história familiar de doença arterial coronariana prematura (para homens abaixo dos 55 anos e para mulheres abaixo dos 65 anos $\left.^{16}\right)$. Os hábitos alimentares, apesar de apresentarem um importante impacto na redução da pressão arterial não foram avaliados por este estudo, considerando a dificuldade logística para aplicação de recordatórios alimentares. Entretanto, foi perguntado a todos os participantes se eles foram orientados pelo médico na última consulta sobre a adoção de hábitos alimentares para controle da hipertensão.

Os participantes também foram questionados sobre o uso de medicamentos anti-hipertensivos, antiagregantes plaquetários, hipolipemiantes e/ou antidiabéticos (ou hipoglicemiantes). Cada medicação identificada foi registrada como seu equivalente genérico. Anti-hipertensivos associados em um único comprimido foram separados em seus componentes individuais. Cada medicação foi classificada dentro de uma categoria de acordo com a sexta edição das Diretrizes Brasileiras de Hipertensão ${ }^{5}$. A soma do número das classes de anti-hipertensivos definiu o número de hipotensores em uso por cada paciente. Essas informações foram aferidas a partir da consulta aos prontuários dos pacientes.

A análise dos dados foi feita através do programa SPSS versão 18. As variáveis coletadas foram apresentadas em forma descritiva, com utilização de média e desvio padrão (dp) para variáveis numéricas e frequência (absoluta e relativa) para variáveis categóricas. A presença das diversas condições foi comparada entre os gêneros por meio do teste qui-quadrado (comparação de proporções) ou teste t de Student (comparação de médias), assumindo-se o nível de significância de 5\% $(\mathrm{p}<0,05)$. A correlação entre o número de fatores de risco e a quantidade de anti-hipertensivos utilizada foi feita pela Correlação de Spearman.

O estudo foi aprovado pelo Comitê de Ética em Pesquisa da Universidade Estadual de Montes Claros (UNIMONTES). Todos os pacientes que participaram do estudo receberam informa- ções pertinentes e assinaram um termo de consentimento livre e esclarecido.

\section{Resultados}

Os hipertensos avaliados foram oriundos de nove municípios da região do norte de Minas Gerais (Bocaiúva, Brasília de Minas, Coração de Jesus, Grão-Mogol, Janaúba, Mirabela, Montalvânia, Montes Claros e São João da Ponte), que foram alocados após o sorteio de 40 unidades de equipes da ESF.

Foram avaliados 505 pacientes nas equipes visitadas. A média de idade da população avaliada foi de 65,5 anos $(\mathrm{dp}=10,3)$ e houve um predomínio de mulheres. A proporção de analfabetos entre o grupo estudado foi de $38,6 \%$ ( $\mathrm{n}=$ 195). Outras características do grupo estudado são apresentadas na Tabela 1, que apresenta também a distribuição dos fatores de risco cardiovascular do EF e o tratamento corrente dos pacientes, com análise comparativa entre homens e mulheres.

Considerando-se todos os fatores de risco avaliados, registrou-se pelo menos uma condição associada em mais de $90 \%$ dos pacientes hipertensos do estudo. A dislipidemia e o sedentarismo predominaram no sexo feminino, enquanto o tabagismo foi mais frequente no sexo masculino. Não houve diferença significativa quanto à presença de diabetes mellitus e à história familiar de doença arterial coronariana quando se comparou os dois sexos (Tabela 1).

Não foi observado fator de risco adicional à hipertensão em 49 (9,7\%) pacientes assistidos pelas ESF. O agrupamento de 3 ou mais fatores de risco esteve presente em $79(15,7 \%)$ pacientes. A maioria absoluta dos pacientes tinha um ou dois fatores de risco adicionais à hipertensão (Tabela 2).

$\mathrm{O}$ uso de anti-hipertensivos foi observado em $481(95,2 \%)$ pacientes da amostra, sendo 317 $(62,8 \%)$ usavam dois ou mais anti-hipertensivos. Tratamento não farmacológico exclusivo foi registrado em 25 (5\%) indivíduos.

Observou-se que usar dois anti-hipertensivos foi o mais frequente, independente do número de agravantes cardiovasculares associados à hipertensão. A Tabela 3 apresenta a distribuição do número de drogas anti-hipertensivas, em relação ao número de fatores de risco. A correlação de Spearman entre o número de anti-hipertensivos e a quantidade de fatores de risco foi de 0,112.

A Tabela 4 apresenta a distribuição do uso de drogas que reduzem o risco cardiovascular con- 


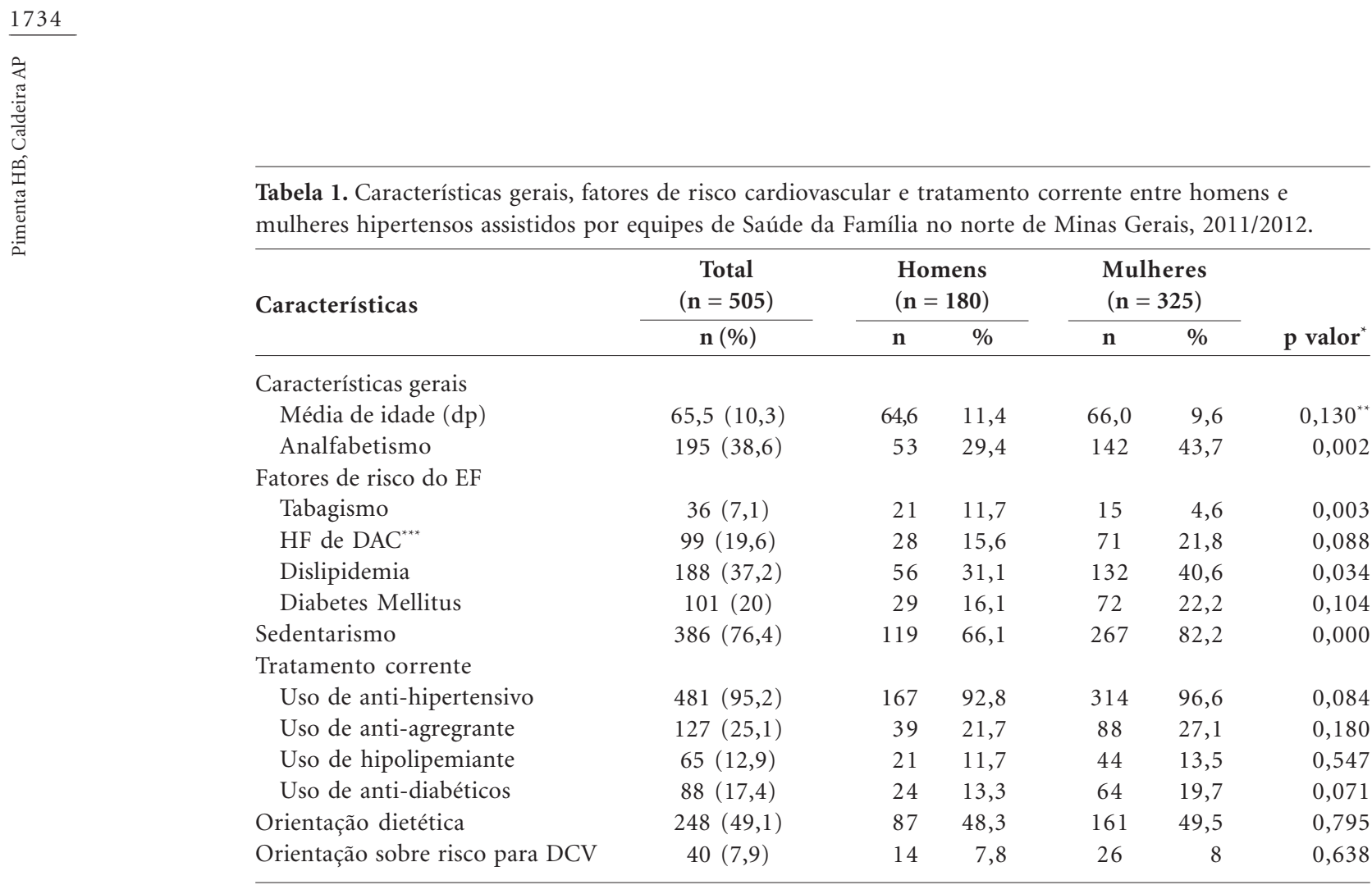

Teste qui-quadrado; ** Teste "t" de Student; ${ }^{* * *}$ História Familiar de Doença Arterial Coronariana.

Tabela 2. Prevalência dos fatores de risco cardiovasculares em homens e mulheres hipertensos assistidos por equipes de Saúde da Família no norte de Minas Gerais, 2011/2012.

\begin{tabular}{crr}
\hline Número de fatores de risco $^{*}$ & $\mathbf{N}$ & $\%$ \\
\hline 0 & 50 & 9,9 \\
1 & 196 & 38,8 \\
2 & 180 & 35,6 \\
3 & 63 & 12,5 \\
$\geq 4$ & 16 & 3,2 \\
\hline
\end{tabular}

*Fatores de risco: Diabetes Mellitus, Dislipidemia, Sedentarismo, Tabagismo, História familiar de doença arterial coronariana.

Tabela 3. Distribuição do número de anti-hipertensivos, conforme a quantidade de fatores de risco cardiovasculares entre hipertensos assistidos por equipes de Saúde da Família no norte de Minas Gerais, $2011 / 2012$.

\begin{tabular}{|c|c|c|c|c|c|c|c|c|c|c|}
\hline \multirow{3}{*}{$\begin{array}{c}\text { Número de } \\
\text { hipertensivos }\end{array}$} & \multicolumn{10}{|c|}{ Quantidade de fatores de risco cardiovascular } \\
\hline & \multicolumn{2}{|c|}{0} & \multicolumn{2}{|c|}{1} & \multicolumn{2}{|c|}{2} & \multicolumn{2}{|c|}{3} & \multicolumn{2}{|c|}{$\geq 4$} \\
\hline & $\mathbf{n}$ & $\%$ & $\mathbf{n}$ & $\%$ & n & $\%$ & $\mathbf{n}$ & $\%$ & $\mathbf{n}$ & $\%$ \\
\hline 0 & 1 & 4 & 16 & 6,4 & 7 & 28 & 1 & 4 & 0 & 0 \\
\hline 1 & 16 & 9,8 & 71 & 43,6 & 54 & 33,1 & 18 & 11 & 4 & 7,8 \\
\hline 2 & 26 & 12,4 & 72 & 34,3 & 76 & 36,2 & 29 & 13,8 & 7 & 3,3 \\
\hline 3 & 5 & 6,3 & 29 & 36,7 & 32 & 40,5 & 11 & 13,9 & 2 & 2,5 \\
\hline$\geq 4$ & 2 & 7,1 & 8 & 28,6 & 11 & 39,3 & 4 & 14,3 & 3 & 10,7 \\
\hline
\end{tabular}

* Teste de Correlação de Spearman (Rho) $=0,112$. 
Tabela 4. Uso de drogas que reduzem o risco cardiovascular conforme o número de fatores de risco associados a hipertensão entre pacientes assistidos por equipes de Saúde da Família no norte de Minas Gerais, 2011/2012.

\begin{tabular}{|c|c|c|c|c|c|c|c|c|c|c|}
\hline \multirow{3}{*}{$\begin{array}{l}\text { Drogas redutoras } \\
\text { do } \mathrm{RCV}^{*}\end{array}$} & \multicolumn{8}{|c|}{ Quantidade de fatores de risco cardiovascular } & & \\
\hline & \multicolumn{2}{|c|}{$\begin{array}{c}0 \\
(n=50)\end{array}$} & \multicolumn{2}{|c|}{$\begin{array}{c}1 \\
(n=196)\end{array}$} & \multicolumn{2}{|c|}{$\begin{array}{c}2 \\
(n=180) \\
\end{array}$} & \multicolumn{2}{|c|}{$\begin{array}{c}3 \\
(n=63)\end{array}$} & \multicolumn{2}{|c|}{$\begin{array}{c}\geq 4 \\
(n=16)\end{array}$} \\
\hline & $\mathbf{n}$ & $\%$ & $\mathbf{n}$ & $\%$ & $\mathbf{n}$ & $\%$ & $\mathbf{n}$ & $\%$ & $\mathbf{n}$ & $\%$ \\
\hline Anti-hipertensivos & 49 & 98 & 181 & 97 & 173 & 96,1 & 62 & 98,4 & 16 & 100 \\
\hline Antiagregante plaquetário & 7 & 14 & 33 & 16,8 & 51 & 28,3 & 27 & 42,9 & 9 & 56,3 \\
\hline Hipolipemiantes & 0 & 0 & 9 & 4,5 & 34 & 18,8 & 16 & 25,4 & 6 & 37,5 \\
\hline Antidiabéticos & 0 & 0 & 10 & 5,1 & 38 & 2,1 & 27 & 42,9 & 13 & 81,3 \\
\hline
\end{tabular}

RCV = Risco cardiovascular.

forme o número de fatores de risco associados à hipertensão para os pacientes estudados.

\section{Discussão}

Os resultados deste estudo mostram que na população hipertensa avaliada houve uma presença expressiva de outros fatores de risco que aumentam a probabilidade de desenvolvimento de um evento cardiovascular adverso. Este achado confere ao Escore de Framingham um destacado potencial para auxiliar os profissionais de saúde da atenção primária a estratificarem o risco cardiovascular da população assistida. Esta estratégia de abordagem estratificada pode modificar o manejo do hipertenso para uma forma mais completa, isto é, levando em consideração não apenas as cifras tensionais, mas também as condições clínicas associadas. Vários estudos ${ }^{9,17-19}$ evidenciam estes achados, demonstrando que a prevenção da morbidade e mortalidade do sistema cardiovascular implica em abordagem estratificada do hipertenso.

O agrupamento de dois ou mais fatores de risco esteve presente em aproximadamente metade dos indivíduos estudados. Proporção semelhante foi relatada por Kannel ${ }^{20}$, que afirma que esta frequência é duas vezes aquela esperada pelo acaso. Estes dados indicam que a pressão arterial elevada, na maior parte das vezes não é apenas uma condição isolada, mas faz parte do perfil do indivíduo de risco cardiovascular elevado ${ }^{21}$.

Estudos recentes mostram que entre os hipertensos idosos há um predomínio do sexo feminino $^{15,22}$. Este dado também foi verificado no presente trabalho. Esta informação é bastante relevante para a população estudada, pois após os 45 anos de idade, os escores no item idade são maiores para mulheres em todas as faixas etárias. Além disso, a presença de diabetes mellitus e os níveis de colesterol têm maior peso no sexo feminino $^{7}$. O predomínio de mulheres no grupo de hipertensos assistidos pelas equipes de saúde da família destaca uma tendência para maiores escores no processo de estratificação e, consequentemente, identificação de riscos cardiovasculares mais elevados.

Além de mostrar uma predominância de mulheres hipertensas, este estudo identificou que alguns dos fatores de risco cardiovasculares são mais frequentes nas idosas. Foi o caso da dislipidemia e do sedentarismo. Apesar da inatividade física não influenciar na escala de Framingham, ela está associada à maior morbi-mortalidade cardiovascular ${ }^{1}$. O exercício físico está associado à redução de doenças, de incapacidades, além de melhorar a qualidade de vida nos idosos ${ }^{23}$. Por outro lado, a alteração do perfil lipídico está diretamente ligada à aterosclerose e suas consequências e tem valor no escore de Framingham. Por exemplo, uma mulher diabética, na faixa etária de 65 a 69 anos e com o colesterol total de 240 $\mathrm{mg} / \mathrm{dl}$, alcançará 3 pontos a mais no EF em relação a um homem que tivesse as mesmas características clínicas. Estes dados também reforçam o potencial de utilização desta ferramenta de estratificação nesta população.

$\mathrm{O}$ único fator de risco que se mostrou mais frequente nos homens foi o tabagismo. Entretanto a prevalência do hábito de fumar foi baixa. A literatura ratifica o achado de baixa frequência de fumantes em indivíduos com faixa etária mais elevada, que pode ser justificada pela maior prevalência de doenças e maior preocupação com a saúde entre as pessoas idosas ${ }^{24}$. 
Os dados obtidos sobre a prevalência de diabetes mellitus e dislipidemia na amostra avaliada, estão em consonância com a literatura científi$\mathrm{Ca}^{15,25}$, que indica uma frequência maior destas condições em indivíduos idosos. Além da faixa etária elevada, o fato da amostra ser de indivíduos com diagnóstico de HAS, que hoje é considerada uma síndrome com vários aspectos clínicos ${ }^{21}$, pode explicar a maior prevalência encontrada de diabetes e dislipidemia. Estes dados reforçam o potencial do EF para esta população, pois pode identificar pacientes de alto risco e que se beneficiariam de um tratamento mais rigoroso.

A estratificação do hipertenso é uma forma de adotar estratégias mais apropriadas no manejo da HAS, conforme o risco projetado para o desenvolvimento de um evento adverso. Estas estratégias envolvem, por exemplo, o uso medicações que podem reduzir este risco, conforme o nível de estratificação. Além disso, pacientes identificados como alto risco cardiovascular e com metas de níveis pressóricos mais baixos devem estar com um tratamento anti-hipertensivo mais intensivo ${ }^{16}$. No presente estudo, essa análise foi feita a partir do coeficiente de Spearman, que varia entre -1 e 1. Quanto mais próximo estiver destes extremos, maior será a associação entre as variáveis. $\mathrm{O}$ sinal negativo da correlação significa que as variáveis variam em sentido contrário. $\mathrm{O}$ que se observou é que houve uma correlação fraca entre o número de anti-hipertensivos e a quantidade de fatores de risco, isto é, o uso de medicações hipotensoras estava pouco ajustado conforme a presença de agravantes de risco cardiovascular associados. A maioria dos hipertensos usava dois anti-hipertensivos. Em uma publicação recente, Egan et al. ${ }^{26}$ mostraram que o uso de apenas 1 ou 2 anti-hipertensivos, a idade avançada e o EF elevado, são variáveis independentes associadas à falta de controle pressórico do hipertenso.

Os pacientes hipertensos e classificados como de alto risco cardiovascular devem estar em uso de medicações adjuntas como aspirina e hipolipemiantes que podem evitar um evento adver$\mathrm{so}^{4,7,16,27,28}$. No presente estudo, a prevalência do uso de drogas que reduzem o risco cardiovascular também foi aferida. Entre os pacientes com três ou mais fatores agravantes associados à HAS, mais de $50 \%$ não estavam em uso de antiagregante plaquetário e mais de dois terços não usava hipolipemiante. Pacientes com este perfil clínico e metabólico somam mais pontos no EF e têm um potencial benefício de utilizar estas medicações. Além disso, pode-se observar que entre os hipertensos com um ou nenhum fator de risco associado, e, provavelmente, com um escore de estratificação baixo, cerca de um quarto estavam em uso de anti-agregante plaquetário (ácido acetilsalicílico), o que deveria ser reservado para pacientes de alto risco ${ }^{27}$. Esses achados revelam uma falta de padronização no acompanhamento e manejo do paciente hipertenso ao nível da atenção primária, para a população estudada. Novamente desvela-se uma oportunidade para a aplicação do EF, pois estratificando o hipertenso, pode-se acrescentar medicações que diminuem a morbi-mortalidade cardiovascular e também evitar o uso de medicações com potencial iatrogênico nos pacientes de baixo risco.

A HAS é responsável por 7,5 milhões de mortes no mundo, cerca de $13 \%$ de todas as mortes anuais. Existe uma projeção de aumento de 15\% a este número na década atual ${ }^{1}$. A prevenção e o controle adequados da HAS podem mudar este cenário. Para isto é necessário que o foco do manejo do hipertenso não seja direcionado apenas para as cifras tensionais. A abordagem deve ser global, considerando os fatores de risco associados. Entretanto o sinergismo desta associação é complexo, o que torna necessário a utilização de instrumentos para o cálculo do risco, para que não haja subjetividade nesta avaliação. $\mathrm{O} \mathrm{EF}$, apesar de estar sujeito a correções conforme indicadores epidemiológicos da população onde é aplicado, identifica adequadamente os indivíduos de baixo e alto risco ${ }^{7,9,29}$. Os dados deste estudo ratificam esta aplicação para a população que foi estudada, pois mostraram uma grande prevalência dos fatores utilizados no EF.

A estratificação de Framingham auxilia não apenas o médico no manejo do hipertenso, mas também pode ajudar a aumentar a participação deste indivíduo no seu tratamento, quando este toma conhecimento do seu risco cardiovascular ${ }^{30,31}$. Os achados deste trabalho mostraram que um número muito pequeno de pessoas avaliadas relatou ter recebido informação sobre a chance de desenvolver um evento cardiovascular. Este fato pode estar associado à alta prevalência de analfabetismo na população avaliada. Níveis educacionais baixos estão associados com dificuldade de percepção das necessidades de controle da $\mathrm{HAS}^{32}$. Porém, a não utilização da tabela do EF pelo médico também pode justificar a falta de comunicação ao paciente sobre o seu risco. Destaca-se, assim, outro papel importante da utilização do EF na atenção primária, que é o de promover o autocuidado e a responsabilidade compartilhada do manejo da hipertensão. Este representa, pois, mais um desafio às equipes da 
ESF, já que parece haver um consenso de que as intervenções de base comunitária e o papel protagonista dos serviços de atenção primária são fundamentais para o sucesso da prevenção integrada dos fatores de risco cardiovascular ${ }^{33}$.

Os resultados do presente estudo devem ser analisados considerando algumas limitações. Apesar de se tratar de uma amostra de base populacional, os indivíduos alocados foram limitados àqueles cadastrados no programa da Estratégia de Saúde da Família, o que pode afetar a generalização dos resultados. Alguns dados foram obtidos pelos relatos dos pacientes e podem estar sujeitos a percepções equivocadas dos mesmos e vieses de memória. Outras informações foram obtidas por busca de informações em prontuário, que não seguem um modelo padronizado de registro. Finalmente, a inferência de que os pacientes teriam um alto risco cardiovascular, foi baseada na quantidade de fatores de risco associados à hipertensão. Porém, no EF a magnitude de alguns fatores (por exemplo: valores do colesterol ou níveis pressóricos) podem influenciar na pontuação final e, consequentemente, no nível de risco.

Deve-se considerar ainda que quando se propõe estratificar pacientes para ajustar seus tratamentos com drogas baseadas em fatores de risco identificados é importante entender que tal estratificação não é conduzida apenas pelo número de fatores de risco ou comorbidades, mas também pelo seu tipo. Ou seja, hipertensos com diabetes são diferentes de hipertensos com dislipidemia, o que implica em dizer que o tratamento deve ser adequado a cada paciente, mas não apenas em relação ao número de comorbidades presentes. Essas limitações, todavia, não devem comprometer a necessária reflexão sobre a necessidade de nova abordagem ao paciente hipertenso na atenção primária. Os resultados registram distorções e equívocos que devem ser atenuados para que melhores resultados sejam alcançados.

Esta investigação evidenciou que entre os hipertensos conduzidos na atenção primária através da estratégia de saúda da família, os fatores de risco cardiovascular utilizados no EF são altamente prevalentes. Estes pacientes parecem não estar sendo conduzidos de forma estratificada. Estes fatos indicam um grande potencial na utilização desta ferramenta de estratificação. Assim a aplicação do EF, conforme preconizado pelo Ministério da Saúde, traz consigo o desafio de criar estratégias de capacitação e educação continuada aos profissionais da atenção primária, no sentido de atingir estratificação e manejo mais adequado da população hipertensa assistida.

\section{Colaboradores}

HB Pimenta e AP Caldeira participaram igualmente de todas as etapas de elaboração do artigo. 


\section{Referências}

1. World Health Organization (WHO). Global status report on noncommunicable diseases 2010. Geneva: WHO; 2011.

2. Tocci G, Valenti V, Sciarretta S, Volpe M. Multivariate risk assessment and risk score cards in hypertension. Vasc Health Risk Manag 2007; 3(3):313-320.

3. Van Eyken EBBD, Moraes CL. Prevalência de fatores de risco para doenças cardiovasculares entre homens de uma população urbana do Sudeste do Brasil. Cad Saude Publica 2009; 25(1):111-123.

4. Mancia G, De Backer G, Dominiczak A, Cifkova R, Fagard R, Germano G, Grassi G, Heagerty AM, Kjeldsen SE, Laurent S, Narkiewicz K, Ruilope L, Rynkiewicz A, Schmieder RE, Struijker Boudier HA, Zanchetti A, Vahanian A, Camm J, De Caterina R, Dean V, Dickstein K, Filippatos G, Funck-Brentano C, Hellemans I, Kristensen SD, McGregor K, Sechtem U, Silber S, Tendera M, Widimsky P, Zamorano JL, Kjeldsen SE, Erdine S, Narkiewicz K, Kiowski W, Agabiti-Rosei E, Ambrosioni E, Cifkova R, Dominiczak A, Fagard R, Heagerty AM, Laurent S, Lindholm LH, Mancia G, Manolis A, Nilsson PM, Redon J, Schmieder RE, Struijker-Boudier HA, Viigimaa M, Filippatos G, Adamopoulos S, Agabiti-Rosei E, Ambrosioni E, Bertomeu V, Clement D, Erdine S, Farsang C, Gaita D, Kiowski W, Lip G, Mallion JM, Manolis AJ, Nilsson PM, O’Brien E, Ponikowski P, Redon J, Ruschitzka F, Tamargo J, van Zwieten P, Viigimaa M, Waeber B, Williams B, Zamorano JL, The task force for the management of arterial hypertension of the European Society of Hypertension, The task force for the management of arterial hypertension of the European Society of Cardiology. 2007 Guidelines for the management of arterial hypertension. Eur Heart J 2007; 28(12):1462-1536.

5. Sociedade Brasileira de Cardiologia, Sociedade Brasileira de Hipertensão, Sociedade Brasileira de Nefrologia. VI Diretrizes Brasileiras de Hipertensão. Arq Bras Cardiol 2010; 95(Supl. 1):1-51.

6. Brasil. Ministério da Saúde (MS). Secretaria de Atenção à Saúde. Departamento de Atenção Básica. Hi pertensão arterial sistêmica para o Sistema Único de Saúde. Brasília: MS; 2006.

7. Wilson PWF, D'Agostino RB, Levy D, Belanger AM Silbershatz H, Kannel WB. Prediction of Coronary Heart Disease Using Risk Factor Categories. Circulation 1998; 97(18):1837-1847.

8. Eichler K, Puhan MA, Steurer J, Bachmann LM. Prediction of first coronary events with the Framingham score: A systematic review. Am Heart J 2007; 153(5):722-731.

9. Zhu B, Haruyama Y, Muto T, Yamasaki A, Tarum F. Evaluation of a community intervention program in Japan using Framingham risk score and estimated 10-year coronary heart disease risk as outcome variables: a non-randomized controlled trial. $B M C$ Public Health 2013; 13:219.

10. Pereira JC, Barreto SM, Passos VMA. Perûl de risco cardiovascular e autoavaliação da saúde no Brasil: estudo de base populacional. Rev Panam Salud Publica 2009; 25(6):491-498.
11. Santos JC, Moreira TMM. Risk factors and complications in patients with hypertension/diabetes in a regional health district of northeast Brazil. Rev Esc Enferm USP 2012; 46(5):1124-1131.

12. Costa JMBS, Silva MRF, Carvalho EF. Avaliação da implantação da atenção à hipertensão arterial pelas equipes de Saúde da Família do município do Recife (PE, Brasil). Cien Saude Colet 2011; 16(2):623-633.

13. Pierin AMG, Marroni SN, Taveira LAF, Benseñor IJM. Controle da hipertensão arterial e fatores associados na atenção primária em Unidades Básicas de Saúde localizadas na Região Oeste da cidade de São Paulo. Cien Saude Colet 2011; 16(Supl. 1):13891400.

14. Instituto Brasileiro de Geografia e Estatística (IBGE). Censo nacional de 2010. [Internet]. [acessado 2013 mar 14]. Disponível em: http://censo2010.ibge. gov.br/apps/atlas/

15. Chamnan P, Simmons RK, Khaw KT, Wareham NJ, Griffin SJ. Estimating the population impact of screening strategies for identifying and treating people at high risk of cardiovascular disease: modelling study. BMJ 2010; 340:c1693.

16. Chobanian AV, Bakris GL, Black HL, Cushman WC, Green LA, Izzo Junior JL, Jones DW, Materson BJ, Oparil S, Wright JT Junior, Roccella EJ; Joint National Committee on Prevention, Detection, Evaluation, and Treatment of High Blood Pressure. National Heart, Lung, and Blood Institute; National High Blood Pressure Education Program Coordinating Committee. Seventh Report of the Joint National Committee on Prevention, Detection, Evaluation, and Treatment of High Blood Pressure. Hypertension 2003; 42(6):1206-1252.

17. Girotto E, Andrade SM, Cabrera MPA, Ridão RG. Prevalência de fatores de risco para doenças cardiovasculares em hipertensos cadastrados em unidade de saúde da família. Acta Sci Health Sci 2009; 31(1):7782.

18. Nascimento ES, Branco MPFC, Moreira AKF, Hazime FA. Estratificação do risco cardiovascular global em hipertensos atendidos numa unidade de saúde da família de Parnaíba, Piauí. Rev Bras Promoç Saúde 2012; 25(3):287-294.

19. Teles CPS, Filho SCT, Sousa ACS, Barreto-Filho JAS. Hipertensão: um estado pró-trombótico. Rev Bras Hipertens 2007; 14(4):245-251.

20. Kannel WB. Risk Stratification in Hypertension: New Insights from the Framingham Study. Am J Hypetens 2000; 13(Supl.):3s-10s.

21. Giles TD. Global cardiovascular risk reduction: new concepts and therapeutic opportunities. J Clin Hypertens 2006; 8(Supl. 8):2-4.

22. Mosca L, Benjamin EJ, Berra K, Bezanson JL, Dolor RJ, Lloyd-Jones DM, Newby LK, Piña IL, Roger VL, Shaw LJ, Zhao D. Effectiveness-Based Guidelines for the Prevention of Cardiovascular Disease in Women 2011 Update: A Guideline From the American Heart Association. Circulation 2011; 123(11):1-20. 
23. Elsawy B, Higgins KE. Physical activity guidelines for older adults. Am Fam Physician 2010; 81(1):55-59.

24. Berto SJP, Carvalhaes MA, Moura EC. Tabagismo associado a outros fatores comportamentais de risco de doenças e agravos crônicos não transmissíveis. Cad Saude Publica 2010; 26(8):1573-1582.

25. McDonald M, Hertz RP, Unger AN, Lustik MB Prevalence, Awareness, and Management of Hypertension, Dyslipidemia, and Diabetes Among United States Adults Aged 65 and Older. J Gerontol A Biol Sci Med Sci 2009; 64(2):256-263.

26. Egan BM, Zhao Y, Axon RN, Brzezinski WA, Ferdinand KC. Uncontrolled and Apparent Treatment Resistant Hypertension in the United States, 1988 to 2008. Circulation 2011; 124(9):1046-1058.

27. Calvin AD, Aggarwal NR, Murad MH, Shi Q, Elamin MB, Geske JB, Fernandez-Balsells MM, Albuquerque FN, Lampropulos JF, Erwin PJ, Smith SA, Montori VM. Aspirin for the Primary Prevention of Cardiovascular Events. Diabetes Care 2009; 32(12): 2300-2306.

28. Seshasai SR, Wijesuriya S, Sivakumaran R, Nethercott S, Erqou S, Sattar N, Ray KK. Effect of Aspirin on Vascular and Nonvascular Outcomes. Arch Intern Med 2012; 172(3):209-216.

29. Khalili D, Hadaegh F, Soori H, Steyerberg EW, Bozorgmanesh M, Azizi F. Clinical Usefulness of the Framingham Cardiovascular Risk Profile Beyond Its Statistical Performance: The Tehran Lipid and Glucose Study. Am J Epidemiol 2012; 176(3):177-186.

30. Vieira AJ, Sheridan SL. Global Risk of Coronary Heart Disease: Assessment and Aplication. Am Fam Physician 2010; 82(3):265-274.

31. Shillinglaw B, Viera AJ, Edwards T, Simpson R, Sheridan SL. Use of global coronary heart disease risk assessment in practice: a cross-sectional survey of a sample of U.S. physicians. BMC Health Serv Res 2012; 12:20.

32. Grotto I, Huerta M, Sharabi Y. Hypertension and socioeconomic status. Curr Opin Cardiol 2008; 23(4):335-339.

33. Ribeiro AG, Cotta RMM, Ribeiro SMR. A Promoção da Saúde e a Prevenção Integrada dos Fatores de Risco para Doenças Cardiovasculares. Cien Saude Colet 2012; 17(1):7-17.

Artigo apresentado em 23/08/2013

Aprovado em 08/10/2013

Versão final apresentada em 18/10/2013 